\title{
Современные тенденции развития конденсаторов
}

\author{
М. Макушин ${ }^{1}$, И. Черепанов²
}

УДК 621.38 | ВАК 05.27 .01

\begin{abstract}
Рынок пассивных компонентов в последнее время находится в неустойчивом состоянии, существуют определенные проблемы с цепочками поставок, и по ряду товарных позиций начинает возникать дефицит, особенно

в отношении таких компонентов, как многослойные керамические конденсаторы и резисторы. Ограниченная база поставщиков материалов может стать значительным фактором риска для поставщиков конденсаторов. Общими тенденциями развития конденсаторов является снижение размеров и улучшение эксплуатационных характеристик.
\end{abstract}

\section{СИТУАЦИЯ НА РЫНКЕ ПАССИВНЫХ КОМПОНЕНТОВ}

Мировой рынок пассивных компонентов и соединительных элементов в 2018 году достиг объема в 168,8 млрд долл. и, вероятно, будет увеличиваться в период с 2019 по 2027 год в среднем на 5,1\% ежегодно. Электронные гаджеты стали неотъемлемой частью жизни каждого человека. Бесконечный спрос на новые и улучшенные потребительские электронные продукты, такие как смартфоны, цифровые камеры, персональные цифровые помощники и т.д., постоянно стимулирует более высокую функциональность в более миниатюрных корпусах. Новые устройства требуют не только ИС, но и все большего количества пассивных компонентов, что связано с увеличением числа функций каждого нового гаджета. В дальнейшем развитие и более широкое внедрение различных потребительских электронных устройств будет способствовать росту мирового рынка пассивных компонентов.

С географической точки зрения наибольшая доля мирового рынка пассивных компонентов в 2018 году пришлась на Азиатско-Тихоокеанский регион. Ожидается, что в период с 2019 по 2027 год его доля только увеличится [1].

Пассивные компоненты широко используются в электронной технике, и их производители, как и производители других типов полупроводниковых приборов, предъявляют спрос на материалы. По данным корпорации Paumanok Publications, стоимость основных материалов, применяемых при производстве пассивных компонентов в качестве диэлектриков, электродов и т. д., в 2019 году составила 10 млрд долл. На конденсаторы приходится до 70\% спроса, 30\% - на резисторы, магниты, датчики и другие компоненты [2].

ЦНИИ «Электроника», главный специалист, mmackushin@gmail.com. цнии «Электроника», главный специалист.

\section{КОНДЕНСАТОРЫ:} НОВОСТИ И ТЕНДЕНЦИИ 2018-2019 ГОДОВ

В настоящее время человечество живет в период перехода в цифровой век. Этот шаг стал возможен благодаря быстрому развитию электроники, особенно вычислительной техники, обеспечивающих совершенно новый уровень сетей и услуг во всех аспектах нашей жизни (табл. 1).

Новый уровень объемов цифровых услуг и сетей обеспечивается новейшим электронным оборудованием, технологиями с высокими вычислительными возможностями, возможностями передачи данных и хранения. Последние платформы, такие как Интернет вещей, промышленный Интернет вещей (в рамках 4-й промышленной революции"), 5G-сети и технологии V2X требуют экспоненциального увеличения глобального трафика данных и мобильных данных, которые должны поддерживаться электронным оборудованием и инфраструктурой. Все это приводит к более высоким требованиям, предъявляемым к электронному оборудованию, архитектуре проектирования плат и пассивным компонентам (табл. 2).

Industry 4.0 (The Fourth Industrial Revolution) - 4-я промышленная революция, ожидаемое массовое внедрение киберфизических систем в производство. Также это производственная сторона, эквивалентная ориентированному на потребителей Интернету вещей. Одним из существенных аспектов Industry 4.0 является идея "сервис-ориентированного проектирования" (от пользователей, использующих заводские настройки для производства собственных продуктов, до компаний, которые поставляют индивидуальные продукты индивидуальным потребителям)

V2X (Vehicle-to-Everything) - обобщенное название технологий связи "автомобиль - автомобиль" (V2V) и "автомобиль - инфраструктура" (V2I), которые являются основным единым решением в среде "подключенного" автомобиля. 
Таблица 1. Изменения в парадигмах вычислительной техники

\section{Смена парадигм сектора вычислительной} техники за 60 лет

Технологии, обеспечивающие цифровизацию

$1960-1980$

\begin{tabular}{ll}
\hline ПК & $1980-2000$ \\
\hline Интернет & $2000-2010$ \\
\hline Мобильные и социальные сети & $2010-2015$ \\
\hline Услуги & $2015-2020$ \\
\hline Искусственный интеллект & $2020-.$. \\
\hline
\end{tabular}

\section{Тенденции рынка и технологии}

Основные тенденции развития рынка и технологий пассивных компонентов - рост инвестиций в разработку новых процессов и материалов для внедрения совершенно нового поколения пассивных устройств. Это также подразумевает инвестиции в производственное оборудование и ноу-хау

Лидером в миниатюризации дискретных пассивных компонентов является технология многослойных керамических конденсаторов (MLCC) для поверхностного монтажа, в 2018 году основным размером корпуса стал тип 0201, а меньшие размеры корпусов, вплоть до 008004 , в настоящее время шаг за шагом осваиваются в производстве бытовой электроники. Коммерциализация конденсаторов в корпусах менее 0201 и создание компонентов с высокой емкостью (> 1000 диэлектрических слоев на кристалл) стали возможными благодаря переходу от технологии толстых пленок к тонкопленочному осаждению.

\section{Рынок MLCC}

В 2018 году наблюдался резкий рост спроса на MLCC, за которым последовали сбои поставок на рынок и рост цен. Благодаря недавнему (середина 2019 г.) снижению спроса на мировом рынке, можно заметить некоторую стабилизацию ситуации с MLCC малых размеров. Большинство поставщиков сообщают о сокращении заявок на бронирование, а некоторые универсальные приборы становятся более доступными. Тем не менее автомобильные MLCC, крупноразмерные MLCC и MLCC высокой емкости остаются дефицитными изделиями и требуют предварительного бронирования заказов. Отмечается, что снятье с производства, но все еще находящиеся в продаже MLCC (товарно-материальные запасы) по-прежнему ограничивают рост спроса на новые приборы. Производство MLCC автомобильного класса будет полностью распланировано

Таблица 2. Основные последствия цифровизации (включая развитие платформ Интернета вещей, промышленного Интернета вещей, 5G-сетей и средств связи, технологий V2X) для требований к электронному оборудованию, архитектуре проектирования плат и пассивных компонентов

\section{Последствия для} электронного оборудования

1. Увеличение объемов данных для обработки самим прибором.

2. Переход с РЧ на оптические линии (инфраструктура).

3. Переход от систем на печатной плате к "системам-на-кристалле".

4. Увеличение плотности расположения элементов и интегрированные решения.

5. Управление цифровыми, мощностными, радиочастотными / оптическими интерфейсами на одной и той же плате/блоке, часто с требованиями уменьшения размеров последних.

6. Увеличение рассеиваемой мощности и рабочей температуры.

7. Миниатюризация при сохранении / улучшении производительности системы.

8. Сокращение издержек и времени вывода новой продукции на рынок.
Последствия для архитектуры проектирования плат

1. Переход к широкозонным полупроводниковым технологиям ( $\mathrm{GaN} / \mathrm{SiC})$ для увеличения скорости, частоты и эффективности работы при уменьшении размеров.

2. Обеспечение возможности расширения высокотемпературных приложений за счет $\mathrm{GaN} / \mathrm{SiC}$.

3. Из-за существенного увеличения числа различных функций и типов интерфейсов все большим числом различных уровней напряжений питания придется управлять отдельно.

4. Ужесточение ограничений по электромагнитной совместимости - защита малых цифровых сигналов управления и данных в условиях все более шумных электрических сред.

\section{Последствия для} пассивных компонентов

1. Переход к конденсаторам с низким напряжением, высокой емкостью, малым размером и низким ERS ${ }^{*}$ (наибольший интерес - MLCC ${ }^{* *}$ II класса).

2. Переход к сглаживающим конденсаторам большой емкости, низкого напряжения и низкого ERS.

3. Дальнейшее масштабирование, расширение использования встраиваемых решений.

4. Ужесточение требований к технологии тонкопленочного осаждения.

5. Освоение 2D-/3D-печатных компонентов.

6. Расширение применения сильноточных индукторов.

7. Обязательность защиты от перегрузки шунтирующими резисторами.

8. Улучшение контроля электромагнитного излучения (помех).

\footnotetext{
ERS - эквивалентное последовательное сопротивление.

**: MLCC - многослойные керамические конденсаторы
} 
по заказчикам до начала 2020 года, и, по мнению многих поставщиков, такая ситуация сохранится в течение следующих двух лет.

\section{Тенденции масштабирования}

Согласно данным исследования фирмы Paumanok Research, емкость рынка керамических конденсаторов в настоящее время составляет 17,1 млрд долл., ожидается его дальнейший рост. Чтобы удовлетворить будущий спрос, производственные мощности перенаправляются на меньшие, более экономичные размеры корпусов. Различные производители сообщали, что в 2018 году тип 0201 стал основным размером корпуса MLCC, и ожидается, что он останется на этой позиции до 2023 года.

\section{Продажи крупных MLCC по-прежнему важны}

Несмотря на то, что несколько крупных поставщиков решили ограничить производство ряда линеек конденсаторов из-за низкой рентабельности инвестиций, структура продаж практически не изменилась. Правда, если на мировом рынке ощущается тенденция перехода на корпуса меньшего размера, в Европе спрос на MLCC большого размера по-прежнему остается высоким - на них приходится более 60\% объема продаж в Старом Свете. Их основные потребители - производители автомобильной и промышленной электроники. Фирма Paumanok Research ранжировала продажи конденсаторов по типам корпусов исходя из объемов продаж, исчисленных в в евро (результаты приведены в табл. 3).

\section{MLCC высокой емкости}

К керамическим конденсаторам высокой емкости обычно относят приборы емкостью более 1 мкФ. На рынке электролитических конденсаторов постоянно растет доля приборов большой емкости. Однако база поставок конденсаторов высокой емкости ограничена малым числом производителей.

Таблица 3. Многослойные керамические конденсаторы по уровню ценности. Источник: TSI

\begin{tabular}{lr} 
Тип MLCC (по корпусу) & Ранг \\
1210-1825. Высокая емкость & 1 \\
$(17,6 \%$ от общего объема продаж) & 2 \\
\hline 0603 & 3 \\
\hline 0805 & 4 \\
\hline 1206. Высокая емкость & 5 \\
\hline 0805. Высокая емкость & 6 \\
\hline 0603. Высокая емкость & \\
\hline
\end{tabular}

Наиболее используемым диэлектриком в Европе на сегодняшний день является X7R. С этой точки зрения база снабжения производителей конденсаторов высокой емкости еще более ограничена. Дальнейшее развитие MLCC с использованием X7R занимает много времени и требует значительных инвестиций. Ожидается, что в цепочке поставок MLCC высокой емкости дефицит будет пока сохраняться. В настоящее время наиболее востребованными являются конденсаторы емкостью 2,2; 4,7; 10; 22 и 47 мкФ в сочетании с величиной напряжения. Наиболее дефицитными являются крупные приборы на напряжения 25 и 50 в для автомобильной электроники. Использование таких параметров в новых разработках означает конкурентные преимущества для производителей оборудования, но также и потенциальные проблемы с поставками заказчикам, если источник материалов не обеспечен.

Общие тенденции развития конденсаторов и некоторые характеристики/параметры конденсаторов, «впервые в мире» выпущенных в 2018 году, представлены в табл. 4

\section{ОСОБЕННОСТИ ПРИМЕНЕНИЯ Потребительские приборы}

Необходимость миниатюризации печатных плат, достижения в области полупроводниковых и схемных архитектур практически исключили потребность применения в смартфонах конденсаторов, размер корпуса которых превышает 0402. Требования смещаются в сторону меньших значений емкости, снижения эквивалентного последовательного сопротивления (ESR) и высокой пульсирующей компоненты постоянного напряжения, обеспечивающих потребности процессора. Большинство конденсаторов на плате современных смартфонов - это MLCC класса I или класса II (из-за их возможностей уменьшения размеров).

\section{Аэрокосмическая и оборонная промышленность}

Космическая отрасль претерпевает значительные изменения, переходя от доминирующих до сих пор государственных заказов к частному космическому бизнесу. Коммерческие космические проекты для телекоммуникационных спутников, широкого доступа в Интернет или космических транспортных миссий представляют сегодня более 50\% космического бизнеса. Этот переход дает высокую мотивацию для более быстрого внедрения новейших технологий в космическое оборудование и сокращения фазы оценки и аттестации новых технологий. Радиационная стойкость - это ключевой вопрос, который необходимо решить для активных и пассивных компонентов. В этом плане рассматриваются танталовые и алюминиевые конденсаторы. 
Жидкостные [электролитические] танталовые конденсаторы недавно были аттестованы в США на уровне военных стандартов (MIL) для различных применений. Танталовые полимерные конденсаторы были внесены Европейским космическим агентством (ЕКА) в его перечень предпочтительных изделий (ESA Prefered Part List, EPPL), а их многоанодовая версия с более низким ESR в настоящее время проходит аттестацию.

Алюминиевые конденсаторы с герметичными корпусами пытаются войти в оборонный и аэрокосмический бизнес. Их поставщики заявляют о технических преимуществах по сравнению с танталовыми конденсаторами и меньшей стоимости.

Недавние мероприятия по оценке и аттестации включали изучение использования потенциала суперконденсаторов в космосе и жестких условиях окружающей среды. Суперконденсаторные технологии продемонстрировали свою способность работать в определенных условиях при температуре от -80 до $150{ }^{\circ} \mathrm{C}$.

\section{Автомобильная промышленность}

Автомобильная промышленность является одной из основных целевых областей при разработке пассивных компонентов. Основные сегменты - электроника салона (телематика, информационно-развлекательное оборудование, воздушные подушки + системы безопасности, средства связи, бортовая сеть и т.д.), сфера водителя (помощь при вождении, антиблокировочная тормозная система, защита от статического электричества, системы безопасности и т.д.), моторный отсек (генераторы переменного тока, управление батареями, центральный процессор, датчики системы управления двигателем, инвертеры, стартстопные системы ит.д.)

Новые проблемы обусловлены нормативно-правовым регулированием и требованиями стандартов (выбросы, безопасность), улучшенными коммуникационными платформами, а также все большим распространением электромобилей и гибридных автомобилей, что выдвигает новые конкретные требования.

В автомобильной и бортовой электронике производительность оборудования, такого как перспективные системы помощи водителю (ADAS), растет по мере расширения применения компактных монтируемых деталей, обеспечивающих комфортность интерьеров транспортных средств. Поэтому даже отдельные пассивные компоненты должны соответствовать жестким условиям окружающей среды (диапазон температур, вибрации, влажность), требованиям миниатюризации и высоких эксплуатационных характеристик.

Одной из основных тенденций развития автомобилей является быстрая экспансия средств передачи данных и их обработки, связанная с требованиями увеличения вычислительной мощности. Транспортные средства сами по себе становятся облаками, облачными хранилищами больших объемов данных и суперкомпьютерами. В среде транспортного средства существуют многочисленные требования к средствам связи: с транспортной инфраструктурой (V2I), сетями (V2N), интеллектуальными домами

Таблица 4. Общие тенденции развития конденсаторов и новаторские параметры /характеристики приборов, выпущенных в 2018-2019 годах

\section{Общие тенденции развития}

1. Интеграция в микросхемы.

2. Встраивание.

3. Использование в 3D-структурах.

4. Применение новых материалов и технологий.

5. Оптимизация технологий массово-поточного производства.

6. Создание конструкций, устойчивых к жестким условиям окружающей среды.

7. Расширение диапазона параметров и уменьшение размеров.

8. Увеличение мощности (снижение ESR, ESL $\left.{ }^{*} . ..\right)$.

9. Создание нового поколения тонкопленочных технологий для высокопроизводительных приложений.

10. Сокращение издержек.

\section{Новаторские параметры / характеристики}

1. Наименьший пленочный конденсатор постоянного тока.

2. 100-кВ пленочные конденсаторы.

3. Тонкая пленка на температуры до $150{ }^{\circ} \mathrm{C}$.

4. 1000-мкФ MLCС на 2,5 В.

5. 10-мкФ 100-В MLCС в корпусе 3525.

6. Автомобильные MLCC на $175 / 200^{\circ} \mathrm{C}$.

7. Самый маленький в мире MLCC в корпусе 008004.

8. COC MLCC с рабочей температурой до $200{ }^{\circ} \mathrm{C}$.

9. COC и X7R MLCC в корпусе 0603 и рабочим напряжением 1000 B.

10. Аттестация танталовых полимеров для автомобильных и авиакосмических конденсаторов.

11. Алюминиевые автомобильные конденсаторы с устойчивостью к вибрации до 30С.

12. Тонкий суперконденсатор на напряжение 3 В для литий-ионного источника питания.

13. Растягиваемые (эластичные) и гибкие суперконденсаторы.

14. Суперконденсаторы с рабочей температурой от -80 до $150{ }^{\circ} \mathrm{C}$.

15. Суперконденсатор, аттестованный для космического применения.

\footnotetext{
ESL - эквивалентная последовательная индуктивность.
} 


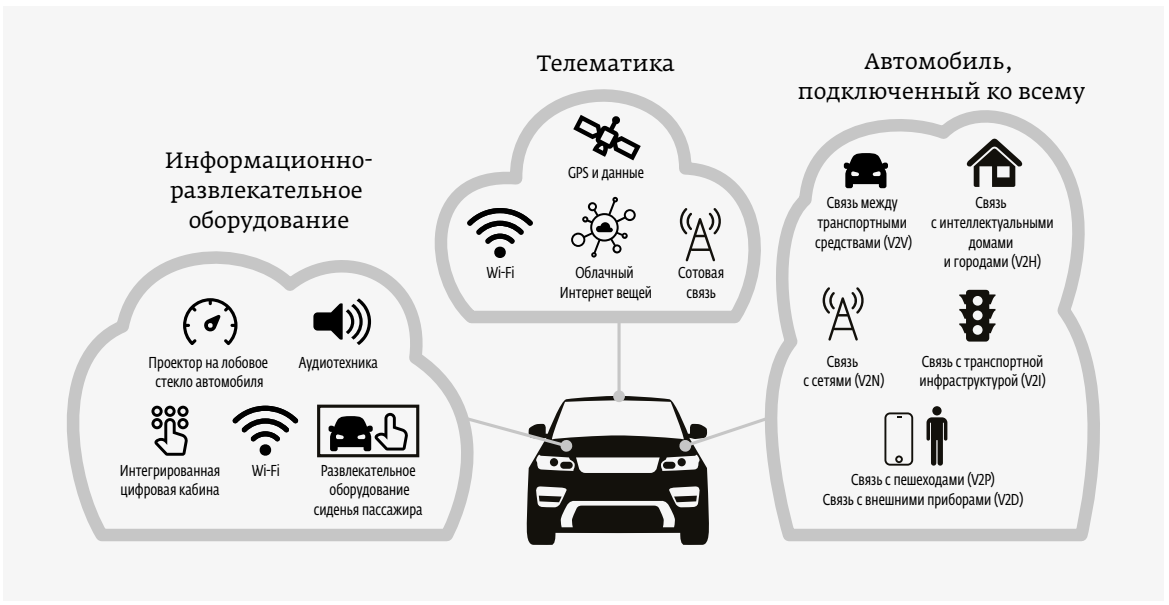

Рис. 1. Среда связи транспортного средства

и городами (V2H), пешеходами (V2P) и между транспортными средствами (V2V). Все эти виды связи с окружающей средой обозначаются термином "автомобиль, подключенный ко всему» (V2X) (рис. 1).

«Подключенные» автомобили и, особенно, следующие за ними автономные (роботизированные) транспортные средства являются "устройствами, подключенными к Интернету вещей», с более высокой скоростью передачи данных, чем смартфон. Это создает новые возможности для сетевых провайдеров. Существует много новых приложений, протоколов и носителей, но их пропускная способность пока ограничена. В течение следующих пяти лет спрос на загрузку данных резко возрастет, при этом скорость загрузки превысит 1 Гбит / с, что значительно увеличит число и сложность радиочастотных элементов. При реализации технологии 5G в диапазоне ниже 6 ГГц будет необходимо обеспечить режим с малым временем ожидания, поскольку автомобиль должен будет сохранить свою автономность в принятии решений. Объем обмена данными автономного транспортного средства может превышать 4 Тбайт / день (рис. 2).

Вышеуказанные требования обуславливают некоторый сдвиг в рекомендациях по выбору технологии компонентов. Пленочные конденсаторы будут заменяться в бортовой электронике на MLCC из-за ограничения размера, срока службы и температуры. В некоторых последних пленочных конденсаторах предельная рабочая температура доведена до $125^{\circ} \mathrm{C}$, тогда как новейшие MLCC функционируют при температуре до 175-200 C, последние также более устойчивы к влажности. С другой стороны, для MLCC проблему представляют вибрации и механическое воздействие.

В автомобильном исполнении теперь доступны танталовые полимерные и алюминиевые электролитические конденсаторы. Первые выдерживают испытания на долговечность по тесту 85/85 в течение
1000 ч (аттестованы по стандарту AECQ-200), а вторые гарантируют устойчивость к вибрациям порядка $30 \mathrm{G}$

Алюминиевые электролитические конденсаторы с полимерным электролитом не пользуются хорошей репутацией из-за проблем с нестабильностью утечки постоянного тока после монтажа платы. Тем не менее, недавно появившаяся "перспективная, гибридная» технология, сочетающая полимер и жидкий электролит, обеспечивает более надежное решение, пригодное для использования в автомобилях. Главное достоинство гибридных алюминиевых конденсаторов - продолжительность срока службы и стабильность параметров изготовления для широкого диапазона автомобильных и промышленных применений.

\section{Промышленная электроника}

Промышленная электроника является широкой областью применения пассивных компонентов - от стандартных условий эксплуатации до условий жесткой окружающей среды с самыми высокими требованиями к надежности работы компонентов. В отличие от автомобильной электроники, где оборудование большую часть срока службы находится в режиме ожидания, или авиакосмической электроники, компоненты которой после сборки работают в условиях отсутствия влажностной и кислородной деградации, промышленные применения часто требуют бездефектной работы всю неделю по 24 ч в сутки на протяжении 10 лет в жестких условиях окружающей среды, таких как низкая / повышенная температура, высокая влажность, повышенное содержание солей в окружающей среде и т.д.

Основными тенденциями в области пассивных компонентов промышленной электроники являются уменьшение размеров, обеспечиваемое использованием "суперматериалов" или "супертехнологий»; расширение диапазона параметров; увеличение устойчивости к жестким условиям окружающей среды и долговечности.

Особый интерес представляют генерация, транспортировка, хранение и сбор энергии от наноразмерных решений на кристалле до высокоэнергетических применений. В первую очередь это касается источников питания электромобилей/гибридных автомобилей, рекуперации", системы высокоэнергетических банков мощности для хранения возобновляемой энергии или сетевого

\footnotetext{
Рекуперация - повторное использование отходов производства или веществ, расходуемых при технологических процессах.
} 


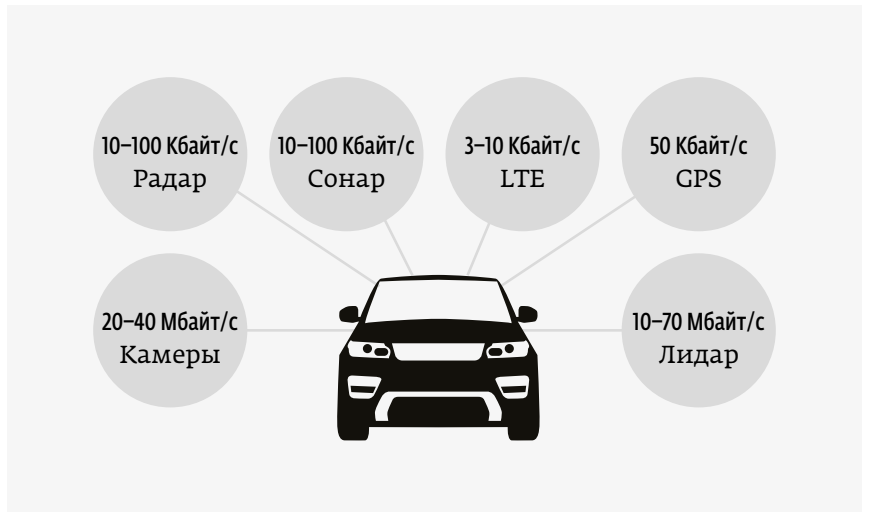

Рис. 2. Объем обмена данными автономного транспортного средства может превышать 4 тбайт / день

хранения и т.д. Последние примеры изделий из этой области включают в себя высокоэнергетические суперконденсаторы с рекордной плотностью энергии хранения, а также разработку новых типов диэлектриков для постоянного напряжения на $\sim 7$ кВ.

\section{ТЕКУЩЕЕ СОСТОЯНИЕ ТЕХНОЛОГИИ И ПЕРСПЕКТИВЫ РАЗВИТИЯ}

Потенциал перспективных разработок для следующих поколений пассивных компонентов можно найти в следующих областях:

хранение энергии в наноразмерном масштабе хранение энергии на кристалле в наномасштабе может быть полезно для небольших кристаллов сбора энергии. Разработки некоторых университетов в области наноразмерных конденсаторов уже нацелены на это приложение;

высоковольтные, высокоэнергетические хранилища - предназначены для рынка электромобилей/гибридных автомобилей, требующих высоковольтных конденсаторов на 400-1000 В с высоким объемным КПД. Такие конденсаторы также требуются для полей генерации энергии из возобновляемых источников (поля ветрогенераторов, солнечных панелей);

тонкопленочные конденсаторы - тонкопленочная технология может быть необходима для удовлетворения потребностей питания носимых датчиков и электроники при обеспечении дальнейшей миниатюризации;

3D-печатные конденсаторы - технология 3D-печати стремительно развивается, открывая новые возможности, недостижимые при использовании современных технологий. Доступные на рынке 3D-принтеры уже могут применяться для печати новых архитектур антенн или датчиков радиочастотной идентификации.

Миниатюризация, высокая скорость передачи данных, увеличение вычислительной мощности, генерация электроэнергии (а также ее передача и хранение) являются основными факторами развития следующего поколения электроники. Эти требования могут вызвать некоторые резкие изменения на рынке в плане разработки и выбора пассивных компонентов. Это может потребовать некоторых улучшений параметров, таких как повышение рабочей температуры, устойчивость к вибрации и жестким условиям окружающей среды, снижение ESR, эквивалентной последовательной индуктивности (ESL) и т.д., что достигается только заменой стандартной технологии конденсаторов, используемой в течение десятилетий.

Крупнейшим сегментом электроники, характеризующимся непрерывным ростом, является потребительская электроника. Однако в "традиционных" сегментах, таких как смартфоны, цифровые фотоаппараты, ПК, ноутбуки, планшетные ПК и т.д., наблюдается падение продаж в натуральном выражении. Самая высокая динамика рынка в настоящее время в автомобильной электронике и в телекоммуникационной / сетевой инфраструктуре, которые сейчас находятся в центре внимания ряда производителей пассивных компонентов. Производители пассивных компонентов также все больше концентрируются на разработке комплексных модульных решений, а не на разработке отдельных компонентов.

Потребностям в дальнейшей миниатюризации и возможности массового производства для поддержки смартфонов и другой портативной электроники наилучшим образом отвечает технология MLCC. Ожидается, что продажи многослойных керамических конденсаторов будут расти в различных секторах промышленности. MLCC с наибольшими размерами корпусов, большей надежностью или рассчитанные на бо́льшую мощность, а также специализированные MLCC являются дефицитным товаром. По прогнозам, спрос будет продолжать расти в течение следующих двух-трех лет.

\section{ЛИТЕРАТУРА}

1. Passive and Interconnecting Components Market Size: expanding at a CAGR of 5.1\% from 2019 to 2027 // Credence Research. Jun 13. 2019.

https://medium.com/@CredenceResearc/passive-andinterconnecting-components-market-size-expanding-at-acagr-of-5-1-from-2019-to-2027-fd4c3813300d

2. Dennis M.Zogbi. Raw Materials or Passive Components: June 2019 Outlook // TTI Inc. November 07. 2019. https://www.ttiinc.com/content/ttiinc/en/resources/ marketeye/categories/passives/me-zogbi-20190711.html

3. Tomáš Zedníček, Martin Bárta, Felix Corbett and Jörg Frodl. Capacitors News and Trends 2018-2019 // Passive Components Blog. 11.27.2019.

https://passive-components.eu/capacitors-news-andtrends-2018-2019/ 